\title{
BIOSSEGURANÇA E PREVENÇÃO DA TUBERCULOSE: A IMPORTÂNCIA DA QUALIDADE DO AR NO INTERIOR DOS SERVIÇOS DE SAÚDE
}

\section{Francelina Helena Alvarenga Lima e Silval e Marli Brito Moreira de Albuquerque Navarro $^{2}$}

\section{RESUMO}

A qualidade do ar nos ambientes dos serviços de saúde onde os profissionais desenvolvem suas atividades está relacionada à construção e manutenção dos sistemas de distribuição de ar. Este é um item relevante do planejamento que inclui a participação dos especialistas em Biossegurança. Como principal recomendação, é importante que tais sistemas restrinjam os agentes de riscos biológicos e químicos que possam ser transmitidos pelo ar a fim de evitar a exposição dos profissionais aos agravos relacionados às enfermidades respiratórias infecciosas. $\mathrm{O}$ conhecimento sobre boas práticas, ventilação, condicionamento do ar; o uso de filtros absolutos e as ações em Biossegurança são fatores determinantes na cadeia de controle de infecções nos serviços de saúde e na manutenção da saúde dos profissionais.

DESCRITORES: Biossegurança; qualidade do ar; tuberculose; saúde do trabalhador.

\section{ABSTRACT}

Biosafety and prevention of tuberculosis: the importance of indoor air quality of health services

The air quality in environments where health care professionals develop their activity is related to the construction and maintenance of air distribution systems as a relevant item of the plan, which includes the participation of experts on Biosafety. As the main recommendation should be noted that systems should restrict agents from biological and chemical risks, which may be transmitted by air to prevent the exposure of professionals to injuries related to respiratory infectious diseases. The knowledge about best practices, ventilation, air conditioning, use of absolute filters and actions on

1 Instituto de Pesquisa Clínica Evandro Chagas (IPEC), Fiocruz. e Centro de Desenvolvimento Tecnológico em Saúde (CDTS), Presidência da Fundação Oswaldo Cruz - Fiocruz, Ministério da Saúde, Brasil.

2 Núcleo de Biossegurança (NUBio), Departamento de Saneamento e Saúde Ambiental(DSSA) da Escola Nacional de Saúde Pública (ENSP), Fundação Oswaldo Cruz- Fiocruz, Ministério da Saúde, Brasil.

Endereço para correspondência: Francelina Helena Alvarenga Lima e Silva, Centro de Desenvolvimento Tecnológico em Saúde (CDTS), Presidência da Fundação Oswaldo Cruz. Avenida Brasil, 4365, Casa Amarela sala 1, Manguinhos, Rio de Janeiro, RJ - Brasil. CEP 21040-900. E-mail: france@fiocruz.br

Recebido para publicação em: 12/4/2012. Revisto em: 23/10/2012. Aceito em: 30/1/2013. 
Biosafety are determining factors in the chain of infection control in health and in maintaining the healthof professionals.

KEY WORDS: Biosafety; air quality; tuberculosis; worker's health.

\section{INTRODUÇÃO}

Estudos referenciais sobre pneumologia destacam a história das doenças ocupacionais associadas à qualidade ambiental. Sublinha-se a importância da qualidade do ar como fator de destaque na identificação de doenças do mundo do trabalho a partir da Revolução Industrial.

$\mathrm{O}$ trabalho de Bernardino Ramazzini, De morbis artificum diatriba $(\mathrm{O}$ Tratado da Doença dos Trabalhadores), de 1700, é a matriz da sistematização da doença ocupacional. Ramazzini, precursor da Medicina do Trabalho, descreveu 54 enfermidades imputadas ao trabalho, às condições e ao ambiente onde os indivíduos executavam suas atividades. Interessou-se pelas doenças que afetavam, principalmente, o proletariado, por isso iniciava com uma entrevista com os pacientes objetivando o diagnóstico da enfermidade que os afetava. Recomendava que se perguntasse: "Qual é a sua profissão?" (27).

Esta pergunta direcionou vários estudos no sentido de estabelecer a relação causal entre as doenças e o desempenho das atividades produtivas, considerando sua natureza, organização e ambiente de trabalho. A relação entre a qualidade do ar e a ocorrência de doenças respiratórias é demonstrada pela interação da superfície do aparelho respiratório, que estabelece a conexão com o meio interno e o ambiente carregado de aerodispersoides (3).

$\mathrm{O}$ aumento das infecções respiratórias e das pneumopatias, assim como seu agravamento, tem sido favorecido pela complexidade e amplitude dos fatores socioeconômicos, culturais, socioambientais e epidemiológicos e da globalização. Como consequências desses fatores, destacam-se as aglomerações humanas (favelas, campos de refugiados), a degradação ambiental, a desnutrição, o deslocamento de populações e as epidemias. Os agentes de risco biológico contribuem para ampliar o quadro por meio da emergência, reemergência e negligência de doenças, em especial as doenças respiratórias infecciosas, sublinhando-se a tuberculose como reemergente em países desenvolvidos. Os agentes de risco biológico colaboram para impactar a sociedade e os governos por meio das doenças emergentes e reemergentes, sobretudo as doenças respiratórias infecciosas, exemplo disso foi o alerta para a probabilidade de uma segunda onda, mais agressiva, do vírus H1N1 (25).

Em 2009, a OMS afirmou que a gripe H1N1 (gripe A) constituía uma preocupação no âmbito da saúde pública mundial e declarou, oficialmente, por meio da mídia e dos órgãos internacionais de saúde, a situação de pandemia do vírus H1N1. A letalidade da doença em alguns países, as dúvidas relativas às informações transmitidas pelos meios de comunicação e o pânico deflagrado contribuíram para 
que milhares de pessoas procurassem os hospitais, clínicas e postos de saúde nos dias críticos da crise. Muitos serviços de saúde e seus trabalhadores naquele e, ainda neste momento, não estão devidamente aparelhados, organizados e capacitados para situações que requeiram resposta imediata em situações de emergência (28).

Neste panorama, inclui-se a tuberculose (TB), doença infecciosa de transmissão direta mediata, isto é por aerossóis, via aérea (pessoa a pessoa). Os trabalhadores dos serviços de saúde desempenham diariamente suas atividades em ambiente de risco, onde ocorrem, frequentemente, atendimentos de pessoas que desconhecem que são portadoras do Mycobacterium tuberculosis. Igualmente, os indivíduos que abandonaram o tratamento representam grande risco. Os trabalhadores de centros de detenção, orfanatos, asilos e hospitais psiquiátricos estão amplamente sujeitos ao contágio. Estes ambientes, especialmente o hospital, podem expandir a perspectiva de infecção pelo M. tuberculosis, uma vez que proporcionam condições de transmissão de um paciente com TB para outro que ocupa o mesmo espaço. Neste caso, destaca-se o atendimento em emergências, enfermarias, unidade de terapia intensiva, etc. Em síntese, estão expostos à TB indivíduos/trabalhadores sadios, como médicos, enfermeiros, técnicos de enfermagem, fisioterapeutas, nutricionistas, dentistas, copeiros, acompanhantes, visitantes, pessoal da limpeza e manutenção e outros prestadores de serviços (22).

Pode-se prevenir a disseminação da TB em serviços de saúde por meio de controle da infecção, obediência às diretrizes de Biossegurança, capacitação e conscientização dos trabalhadores sobre o risco biológico. A TB é uma enfermidade que, apesar de debilitar o paciente, é tratável com o armamento de quimioterápicos disponível desde a metade da Segunda Guerra Mundial. Atualmente, um grande problema é a Tuberculose Resistente a Múltiplas Drogas (MDR-TB). Os indivíduos infectados com linhagens de M. tuberculosis resistentes, ou MDR-TB, são tratados com esquemas diferenciados dos fármacos utilizados para a TB (26).

\section{CONTROLE DO CONTÁGIO DA TUBERCULOSE. INICIATIVAS DOS PROFISSIONAIS DE SAÚDE}

Entre os séculos XVIII e XIX, o contexto socioeconômico foi um fator importante na epidemiologia da doença. Deve-se ressaltar aqui o registro de tuberculose associada ao contexto da industrialização, que favoreceu o inchamento das cidades projetando para os trabalhadores condições de vida e trabalho insalubres. As condições de vida degradantes abaixavam o nível de resistência do indivíduo, o que gerava aumento da predisposição às infecções (14).

O século XIX foi marcado por iniciativas científicas dos mais importantes centros de pesquisa europeus. Estes centros passaram a investir nas pesquisas voltadas para responder às graves questões de saúde pública, buscando, sobretudo, recursos profiláticos tidos como mais eficazes para o controle de epidemias. As transformações geradas pela "era industrial", interferiram no sistema de valores 
da sociedade europeia, favorecendo a perspectiva do higienismo físico e moral, voltado, especialmente, para o controle de doenças entre o proletariado. A compreensão da enfermidade produziu ideias relacionadas ao modo e à qualidade de vida do indivíduo, culpando-o pelo adoecimento (15).

No final do século XIX, as pesquisas de Pasteur e Koch ampliaram o conhecimento da microbiologia. A descoberta de Koch, em 1882, estabeleceu uma nova percepção da tuberculose, destacando-se as investigações sobre infecção, contágio e disseminação. $\mathrm{O}$ controle do contágio incluiu a proteção dos médicos e outros profissionais. A descoberta do bacilo constituiu uma nova visão etiológica e estabeleceu recomendações que alertavam para o caráter biológico da cadeia de transmissão. Até o aparecimento da vacina BCG, oriunda das pesquisas de Calmette e Guérin, o controle do contágio e o tratamento da doença tinham como base as especificações referentes ao ambiente físico e social, observando-se a boa circulação do ar nos ambientes e sua qualidade. A medicina avaliava que o risco de contágio estava presente nas habitações, no ambiente de trabalho, nos hospitais, nos sanatórios, etc. Para serem considerados salubres, esses espaços deveriam ser ventilados e claros, pois umidade, ambientes sombrios e pouco ventilados favoreciam o aparecimento da enfermidade (15).

Também os diversos conflitos bélicos entre os países da Europa aumentaram as condições de transmissibilidade das doenças infecciosas. Na primeira metade do século XIX, destaca-se a Guerra da Crimeia (1853 a 1856), que envolveu as potências europeias, notadamente a França e a Inglaterra. $\mathrm{O}$ conflito resultou num expressivo número de mortos pela elevada incidência de enfermidades infecciosas. O ambiente higiênico sanitário no campo era inimaginável e a mortalidade entre os combatentes feridos ou enfermos era elevadíssima nos hospitais de campanha, ficando a taxa de óbitos em torno de 50\% (16).

Nesse período, o trabalho da enfermeira Florence Nightingale, utilizando medidas radicais, estabeleceu ações de controle de infecções hospitalares. Na volta à Inglaterra, propôs uma reforma hospitalar com base na ordem e higiene dos ambientes, incluindo a separação dos indivíduos pelo tipo de enfermidade. Sua grande contribuição foi estabelecida com a proposta de modificação da arquitetura hospitalar que introduzia maior circulação de ar nas enfermarias. Os princípios que Nightingale usou para administrar e reorganizar o hospital propiciaram aos enfermos condições preventivas que evitavam o agravamento da doença e favoreciam, igualmente, a restauração orgânica e a minimização das infecções (16).

Essas ações representaram uma inovação do ponto de vista epidemiológico das doenças infecciosas e das infecções hospitalares em um tempo pré-pasteuriano e pré- listeriano. O controle das infecções baseado na lavagem das mãos, a higiene corporal e ambiental, a individualização da atenção ao doente, a diminuição do número de leitos por enfermaria e o controle de pessoas alheias ao trabalho hospitalar foram ações empreendidas para o controle sanitário que exerceram impacto na saúde dos indivíduos. Na atualidade, essas ações continuam a ter reflexos, embora 
as doenças se manifestem com novas configurações. O ambiente interno do hospital caracteriza-se como fonte de transmissão de infecção do ambiente para os pacientes e entre eles próprios. A prevenção direcionada a este ambiente ajudou a reduzir a infecção hospitalar e a ampliar o esforço de intervenção no seu controle, tornando o ambiente hospitalar mais seguro (18).

No entanto, a formalização das atividades da enfermagem gerou uma nova classe de trabalhadoras que exerciam a função de enfermeira visitadora e assistente social, expondo-se às diversas doenças comuns na época, principalmente a TB (22). A respeito do risco de contágio para essas profissionais de saúde, no final do século XIX foi realizada uma pesquisa sobre a tuberculose em Londres. A pesquisa não considerou a TB como uma doença contagiosa que pudesse causar risco para enfermeiras, enfermeiras visitadoras, médicos e outros trabalhadores que tivessem contato diretamente com os pacientes. Com a construção dos Sanatoria, os doentes eram afastados do convívio social, mas passavam a constituir um grupo em isolamento que oferecia alto risco para os trabalhadores de saúde. Em razão do isolamento a que os pacientes eram submetidos, diversos estudos sobre os mecanismos e a epidemiologia da doença foram desenvolvidos, o que ajudou a expandir o conhecimento sobre ela. Estudos realizados na primeira metade do século XX em faculdades de enfermagem e medicina, nos Estados Unidos, sobre as taxas de conversão ao teste tuberculínico revelaram informações expressivas, mas estas não foram levadas em conta, negando-se que a possibilidade de infecção dos trabalhadores fosse maior que a da população. Entre 1940 e 1960, a tuberculose foi admitida pela justiça dos Estados Unidos como um agravo do trabalho em instituições de saúde, passando-se a empregar a expressão tuberculose ocupacional para nomear o acometimento dos indivíduos que se infectaram no exercício de atividades laborais nos Sanatoria (22).

Com o advento dos antibióticos como drogas eficazes para combater a TB, após os anos 1950, o risco da TB ocupacional caiu em consequência da menor incidência da doença na população. Após a década de 1970, o tratamento realizado em sanatórios passou a ser feito em hospitais graças ao baixo contágio proporcionado pelo uso dos diversos antibióticos disponíveis. Na década seguinte, 1980, com a associação de drogas (isoniazida, rifampicina e pirazinamida), estabeleceu-se a terapêutica de curta duração realizável no domicílio do paciente. Entre os profissionais de saúde houve uma diminuição da conversão tuberculínica, chegando-se a acreditar que esses trabalhadores não estariam submetidos ao risco biológico proporcionado por este microorganismo (22).

Este quadro foi verificado em países desenvolvidos, mas não se tem muitos dados do que ocorreu em países em desenvolvimento no mesmo período. No Brasil, um estudo realizado nos anos 1970, em São Paulo, demonstrou que os trabalhadores em instituições de saúde tinham quatro vezes maior probabilidade de adquirir a enfermidade do que a população em geral (22). Na metade dos anos 1980, houve uma modificação significativa no quadro da doença. Com o 
aparecimento de pacientes HIV coinfectados com TB, eclodiu uma nova epidemia de TB (31). A infecção pelo HIV alterou a tendência epidemiológica da tuberculose, assim como o aspecto clínico, o tempo de tratamento, etc. (20). O aparecimento da multirresistência aos medicamentos específicos contra a TB se deu nos países desenvolvidos de forma primária com transmissão institucional, incluindo-se a coinfecção pelo HIV, mas se destacam também os casos encontrados por migração. No Brasil, a multiplicidade dos casos de TBMR é pós-primária ou adquirida, não sendo relacionada à coinfecção pelo HIV ou a surtos institucionais. Os surtos de TBMR estão relacionados ao abandono do tratamento e à sua irregularidade (29).

\section{A TUBERCULOSE E A QUALIDADE DO AR INTERIOR NOS SERVIÇOS DE SAÚDE}

Os países desenvolvidos alteraram as práticas de controle de infecção nos hospitais após o início do uso de antibióticos. Ante o crescente avanço farmacológico, as diretrizes e práticas foram substituídas pelo método medicamentoso como alternativa privilegiada da cura da TB. Os recursos destinados ao controle e pesquisa da TB nos Estados Unidos foram reduzidos de maneira drástica, de tal modo que, no início dos anos 1990, apenas 10\%, aproximadamente, dos recursos distribuídos pelas comissões de fomento para pesquisa e desenvolvimento tecnológico foram dirigidos para a área de TB (23).

Em virtude da mudança do quadro epidemiológico da TB, em 1990 foi publicado, nos Estados Unidos pelo Center for Disease Control and Prevention (CDC), um guia para a prevenção da TB nos serviços de saúde e entre os profissionais, com foco nos pacientes com HIV. Foi o primeiro documento que reconheceu a transmissão em estabelecimentos de saúde, com ênfase nas instalações, além de recomendar medidas de proteção como: prevenção de geração de bacilos viáveis, identificação e tratamento da TB ativa, métodos ambientais e de engenharia para controle de aerossóis nos ambientes, uso de equipamento de proteção individual, principalmente dos respiradores com filtros de alta eficiência para os profissionais e vigilância epidemiológica dos serviços de saúde.

Em 2005, os conceitos sobre instalações para atendimento de TB foram ampliados para qualquer relacionamento físico ou organizacional, no qual os trabalhadores dos serviços de saúde partilhassem ambientes com pessoas diagnosticadas com TB ou tivessem contato com amostras clínicas. Vários tipos de configuração de ambientes foram representados, compreendendo áreas como as hospitalares, ambulatoriais e outras não tradicionais. Nas áreas hospitalares, destacam-se: quartos, emergências, unidades de terapia intensiva, salas de cirurgia, laboratórios, sala de broncoscopia, sala de indução do escarro ou salas de terapia de inalação, assim como a sala de autópsia. As áreas ambulatoriais abrangem instalações para tratamento da TB como: consultórios médicos e de cuidados ambulatoriais, unidades de diálise, consultórios dentários, etc. Outros serviços que 
direta ou indiretamente estão relacionados com atendimento de saúde incluem serviço de saúde de atenção de longo e curto prazo (hospícios e abrigos para os sem-teto), unidades correcionais, além de cozinhas, lavanderias, etc. (17).

As medidas tomadas pelos gestores e aquelas incorporadas pelos trabalhadores por meio da capacitação em boas práticas e em Biossegurança para o controle do risco ambiental, na ação contra a TB, quebram os elos da cadeia de transmissão da doença em serviços de saúde. As medidas administrativas, como busca ativa, diagnóstico, terapêutica e acompanhamento do doente se unem às medidas ambientais ou de engenharia que incluem os quartos de isolamento com ventilação natural, os quartos com pressão negativa e o uso de filtros absolutos. As medidas de Biossegurança em todos os níveis envolvem: a recepção do paciente, o diagnóstico laboratorial e o tratamento, a instituição e uso de barreiras primárias como os EPIs pela equipe de trabalhadores, destacando-se, como exemplos desses equipamentos, o respirador com filtro absoluto ou PFF2 (padrão brasileiro e europeu), ou N95 (padrão americano) e a máscara usada pelo paciente (24). O fornecimento e o uso dos EPIs completam as medidas de proteção respiratórias dos trabalhadores na área da saúde. Como medida acessória que fundamenta o controle da TB e atende à NR-32 do TEM, pode ser citado o uso de óculos de proteção, luvas, jaleco ou capote descartável (7).

Outro fator de risco biológico são as amostras obtidas para baciloscopia. Potencialmente contaminadas com micobactérias, devem ser coletadas, transportadas e processadas em ambientes que diminuam o risco de infecção aérea. A dimensão do risco está relacionada com a classe de risco do microrganismo e com a complexidade dos métodos utilizados no processamento e análise da amostra. Como a TB é causada por um microrganismo da classe de risco biológico 3, é de elevado risco para o profissional que o manipula ou para aquele que tem contato direto com pacientes infectados, ao mesmo tempo é considerado de risco moderado para a comunidade no entorno laboratorial. Faz parte do grupo de agentes que são transmitidos por via respiratória e ocasionam enfermidades em humanos e animais. Essas enfermidades, se não forem prevenidas por meio de imunobiológicos ou tratadas com o arsenal de drogas disponíveis, podem levar os indivíduos acometidos à morte. A TB representa risco se disseminada pelos grupos sociais nos diversos tipos de ambiente, uma vez que tem a capacidade de se alastrar entre os indivíduos (9).

No trabalho laboratorial, o $M$. tuberculosis é classificado como agente da classe de risco biológico 3, portanto recomenda-se o processamento de materiais clínicos que não produzem aerossóis nos laboratórios com nível de Biossegurança 2 (NB-2); os que produzem aerossóis devem ser processados em cabine de segurança biológica (CSB) e o trabalhador deverá usar EPI. A análise, a manipulação de culturas, os testes de susceptibilidade às drogas e o crescimento do M. tuberculosis em meio de cultura líquido ou pesquisa devem ser realizados em laboratório com nível de Biossegurança 3 (NB-3). O NB-3 deve possuir instalações, dispositivos, equipamentos de proteção individual e coletiva mais seguros e especializados, bem 
como pressão de ar diferencial, climatização e qualidade do ar interior de acordo com o padrão exigido para essa classe de risco biológico $(8,10)$.

As instalações dos serviços de saúde devem ser planejadas de tal forma que restrinjam os agentes de riscos biológicos e químicos capazes de serem transmitidos pelo ar. É importante ressaltar que a qualidade do ar é um dos fatores determinantes na cadeia do controle de infecções, não se deve, contudo, negligenciar as demais medidas relacionadas às boas práticas e medidas de rotina operacional dos serviços de saúde. Diversos microrganismos foram analisados, ficando demonstrado que causavam, significativamente, infecções em serviços de saúde que possuíam climatização. Dentre esses microrganismos, destacaram-se: Aspergillus, Legionella, Clostridium, Nocardia, Acinetobacter, Serratia, esporos de fungos, vírus e protozoários. O pólen, do mesmo modo, pode ser considerado um contaminante biológico, assim como os ácaros e seus derivados e o M. tuberculosis proveniente da tosse, de espirros, da fala e de aerossóis depositados em partículas no ar. O S. aureus de transmissão direta, por contato, ou indireta, por aerossóis, secreções, poeiras, fômites e alimentos, também está presente na pele, no nariz, no sangue, na urina de pacientes infectados e nas mãos de trabalhadores de saúde, que são fontes de infecção $(1,30)$.

No Brasil, a qualidade do ar interior passou a ter prioridade e relevância em virtude da morte do ministro Sérgio Mota, em 1998, por complicações respiratórias. Essas complicações foram associadas a fatores relacionados à debilidade de sua saúde agravados pelo ambiente onde trabalhava, cujo sistema de ar refrigerado era mal conservado e disseminava ar contaminado. Considerou-se que o ministro foi vítima da Síndrome dos Edifícios Doentes (SED) ou de doença relacionada às edificações. O então ministro José Serra, após estudos realizados por peritos na área, assinou e publicou a Portaria do Ministério da Saúde no 3.523/GM, de 28 de agosto de 1998, que estabeleceu normas, periodicidade para higiene, conservação, operação e controle dos sistemas de climatização. Esta portaria não se aplicava a estabelecimentos de saúde (21).

AAgência Nacional de Vigilância Sanitária (ANVISA), em 24 de outubro de 2000, publicou a Resolução ANVISA/RE n ${ }^{\circ}$ 176, cuja orientação técnica determinava modelos de referência de qualidade de ar interior em ambientes climatizados por equipamentos, tanto de uso público quanto de uso coletivo (5). E em 16 de janeiro de 2003, a ANVISA publicou a Resolução ANVISA/RE n ${ }^{\circ} 9$ que considerava a necessidade de atualizar as orientações publicadas na Resolução ANVISA/RE no 176 . Ambas não mencionavam o uso de climatização artificial em unidades de atendimento à saúde (6).

A norma oficial adotada para condicionamento de ar em unidades de atendimento à saúde é a Norma Brasileira Registrada (NBR) nº 7256 de 1982, da Associação Brasileira de Normas Técnicas (ABNT), que fundamenta as condições mínimas para planejamento e implantação de instalações de tratamento de ar em estabelecimentos assistenciais de saúde (EAS). A segunda edição da NBR 7256, da 
ABNT, em 30 de março de 2005, define os requisitos mínimos para o tratamento de ar com uma classificação de risco ambiental, adota a classificação dos filtros finos utilizados nos equipamentos de climatização. Baseia-se na contagem de partículas, estipula requisitos contra incêndio em instalações de tratamento de ar, assim como determina as providencias a serem estabelecidas na ocorrência de obras ou reformas nos recintos das EAS. A NBR 7256 (2) estipula que os estabelecimentos de saúde devem prover e dimensionar o controle de temperatura, umidade relativa, circulação e grau de pureza do ar, assim como a percentagem de partículas e volume de ar por indivíduo, além do nível de ruído dos equipamentos.

Os equipamentos de climatização e as áreas consideradas como de risco nos serviços de saúde devem ser avaliados por profissionais qualificados com a finalidade de constatar as condições de ventilação e qualidade do ar oferecido aos trabalhadores e clientes. As áreas de maior risco para a TB são as áreas pouco ventiladas, como ambulatórios, sala de espera para atendimento de pacientes com TB, quartos para precaução de transmissão aérea (isolamento respiratório), salas de broncoscopia e escarro induzido, setores de emergência e radiologia e sala de necropsia. Se não atender fisicamente às diretrizes de construção de um laboratório nos níveis de Biossegurança 2 e 3 e outras normas relacionadas a instalações, EPI, EPC e procedimentos, o laboratório de bacteriologia será, igualmente, uma área de risco expressivo para os laboratoristas. Os ambulatórios e salas de espera para atendimento de clínica médica, doenças infecto-parasitárias e pneumologia são potencialmente espaços de risco na transmissão da TB entre indivíduos que nelas permanecem ou transitam (12).

Além dos poluentes microbiológicos, nos serviços de saúde são encontrados aqueles também presentes no ar de edifícios comerciais, locais públicos e industriais como: dióxido de carbono ( $\mathrm{CO} 2)$, monóxido de carbono $(\mathrm{CO})$, dióxido de nitrogênio (NO2), ozônio(O3), formaldeído $(\mathrm{CH} 2 \mathrm{O})$ e compostos orgânicos voláteis totais (TVOC), partículas suspensas respiráveis (RSP), radônio e outros. Em hospitais e outros serviços de saúde são encontrados: glutaraldeído (C5H8O2), óxido nitroso (N2O), partículas alergênicas do látex, contaminação aérea por microrganismos de diâmetro de 1 a $5 \mu \mathrm{m}$ em suspensão no ar, o que permite uma possível infecção aérea. É fundamental para a diluição dos poluentes do ar interno contaminado a introdução de ar externo limpo e em quantidade suficiente para o interior do aparelho de ar-condicionado (19).

$\mathrm{O}$ limite relativo às necessidades de ventilação do ambiente se modifica de acordo com o tipo de ventilação, por exemplo, ar recirculado em relação ao ar fresco. Há duas formas de se medir a taxa de ventilação: a) o volume do espaço, por exemplo, verificado por medida de trocas de ar por hora; b) o número de pessoas em um espaço (litros/segundo/pessoa). A medição baseada em taxas de ocupação e ventilação avalia que cada pessoa em um determinado espaço deve ter uma quantidade de suprimento de ar fresco. Em relação à TB, nos quartos onde não há isolamento, a taxa de ventilação inferior a duas trocas de ar por hora 
está associada às taxas de conversão tuberculínica mais altas. A taxa de ventilação maior ajusta a diluição no ar de microorganismos patogênicos, portanto diminui o risco de infecções aéreas. A OMS recomenda, por precaução, 12 trocas de ar por hora em uma sala, o que equivale a $80 \mathrm{~L} / \mathrm{s} /$ paciente para uma sala de $24 \mathrm{~m}^{3}(32)$. A Resolução ANVISA/RE n 176 recomenda que as taxas de troca de ar externo e total nas principais instalações sejam medidas com base na troca de ar por hora, isto é, a taxa de renovação de ar de $27 \mathrm{~m}^{3} / \mathrm{h} /$ pessoa (5). Para as áreas críticas, como salas de cirurgia e parto, recomendam-se fluxo de ar unidirecional com entrada de ar pelo teto e saída pelo piso e ventilação superior ou igual a 15 trocas de $\mathrm{ar} / \mathrm{h}$. E ainda o controle de temperatura, umidade, pressão, trânsito, número de trabalhadores, manutenção e limpeza do sistema de ar desse ambiente (1).

Fatores importantes na ventilação dos serviços de saúde que devem ser levados em consideração são as condições locais que envolvem construção, estrutura, cultura, clima, regulamentos, custos e qualidade do ar exterior. A ventilação pode ser feita de duas maneiras: ventilação natural e ventilação mecânica. A primeira, mediante ventilação simples e natural, proporciona conforto térmico, renovação do ar e depende da força dos ventos e da temperatura e pode ser otimizada maximizando-se o tamanho da abertura de janelas e localizando-as em paredes opostas. Desse modo, faz-se necessário o estudo das condições climáticas para verificar se essas favorecem o uso do sistema de ventilação natural. A segunda, a ventilação mecânica, por ser sistema bem concebido com operação e manutenção constantes, pode ajudar na diluição adequada, uma vez que a ventilação natural sem a ajuda da mecânica não pode fornecer taxas de ventilação satisfatórias. Em alguns ambientes, a ventilação mecânica (com ou sem controle de temperatura) pode ser necessária, por exemplo, onde o sistema de ventilação natural é insuficiente em razão das condições locais, como a estrutura do edifício, o clima e a qualidade do ar exterior (32).

Nos laboratórios NB-2 e NB-3 (laboratórios de contenção), as Cabines de Segurança Química (CSQ) e as de Segurança Biológica (CSB) são barreiras físicas para as emissões de agentes de risco. As CSB, igualmente, devem ser usadas como obstáculo à dispersão das fontes críticas de contaminantes em preparações e procedimentos realizados. Atenção especial deve ser dada às manipulações que geram aerossóis nas salas de esterilização, de endoscopia, de processamento de tecidos, etc. O risco à saúde dos trabalhadores, pacientes e outros indivíduos que frequentam os serviços de saúde pode ser aumentado pela exposição ao ar interior, possivelmente contaminado. A NBR 7256 classifica o risco de ocorrência de acontecimentos desfavoráveis à saúde por exposição ao ar ambiental em: nível zero - no qual o risco é muito baixo e não excede aquele encontrado em ambientes públicos e coletivos; nível 1 - não existe evidência de agravo à saúde, mas as autoridades sugerem que o risco seja considerado; nivel 2 - há fortes evidências de risco à saúde de pacientes e outros; nível 3 - é elevado o risco de agravos à saúde por causa da qualidade do ar (32). 
A filtração do ar nos ambientes dos serviços de saúde está relacionada com os procedimentos ali realizados. Esta filtração é efetuada por filtros classificados em grossos, finos e absolutos (32). Os filtros removem partículas contaminantes, incluindo microrganismos patogênicos. Múltiplos tipos de filtro podem ser usados para alcançar diferentes graus de limpeza do ar. Instalações de saúde requerem um sistema composto por pré-filtro e filtro final. O pré-filtro deverá remover de $25 \%$ a $30 \%$ da poeira local antes da refrigeração ou aquecimento. Para se conseguir um ar de qualidade, devem ser removidas as grandes partículas do meio, desse modo é alcançada a transferência de calor de forma limpa. O filtro final deve ter $90 \%$ de eficiência para coletar esporos de fungos de diâmetro de $2 \mu \mathrm{m}$ a $5 \mu \mathrm{m}$ ou bactéria que constitua Unidade Formadora de Colônia (UFC) com $1 \mu \mathrm{m}$ de diâmetro ou comprimento. Nas áreas em que se encontrem pacientes imunocomprometidos, é indicado o uso de filtros absolutos ou High-Efficiency Particulate Air (HEPA) que possuem $99,97 \%$ de eficiência sobre partículas de $0,3 \mu \mathrm{m}$ ou, igualmente, o uso de filtros Ultra-Low Pentration Air (ULPA) que possuem 99, 999\% de eficiência sobre partículas de $0,1 \mu \mathrm{m}$ a $0,2 \mu \mathrm{m}$ (19).

O sistema de tratamento controla a qualidade do ar interior e tem a finalidade de reduzir a concentração de partículas e de agentes patogênicos. Este sistema deve evitar fluxos de ar indesejáveis entre os ambientes dos serviços de saúde, mantendo gradientes de pressão interna na direção dos mais limpos para os mais contaminados. $\mathrm{O}$ ambiente de risco deve ser pressurizado com pressão negativa, ou seja, o fluxo de ar é direcionado para fora do referido ambiente. Um diferencial de pressão em relação aos ambientes contíguos se alcança insuflando uma quantidade de ar maior ou menor que a retirada por meio mecânico, simultaneamente para pressão positiva ou negativa. Assim sendo, todos os parâmetros exigem monitoração para garantir que pequenas alterações no desempenho do equipamento não prejudiquem todo o sistema. Ambientes como quarto de isolamento, sala escura, sala de esterilização de equipamentos e laboratórios devem ter pressão negativa. Porém, unidades de terapia intensiva (UTI), sala de isolamento de proteção, sala de cirurgia, sala de parto e farmácia devem ter pressão positiva. O quarto hospitalar comum deve ter pressão igual tanto de entrada quanto de saída do ar. A pressão positiva é obtida quando a inserção de ar em um ambiente é maior do que a retirada, o ar tenderá a sair pelas frestas de portas e janelas $(19,13)$.

Quando o ar contaminado tem origem em salas de tratamento ou em áreas de isolamento e é recirculado, ele deve ser filtrado antes de ser lançado no ambiente. Medidas de precaução devem ser estabelecidas para que este ar não seja lançado no sistema de ventilação geral da instalação. $O$ custo de instalação do sistema de ar se torna mais dispendioso, todavia reduz o risco de contaminação de TB e outros microrganismos para áreas adjacentes. $\mathrm{O}$ ar proveniente de salas de tratamento ou quartos de isolamento de pacientes com TB deve ser exaurido para o espaço exterior e, de maneira nenhuma, recirculado. Em situações nas quais a arquitetura do ambiente impede a exaustão externa, esse ar deverá ser tratado por meio de 
filtração (filtro HEPA) ou irradiação (luz UV) em sistema fechado. Nos isolamentos respiratórios e outros ambientes, o percentual de trocas de ar tem uma variação de 6 a 12 trocas de ar por hora. Recomenda-se que o quarto de isolamento respiratório tenha uma antecâmara com pressão maior que a dos ambientes próximos. A antecâmara promove a contenção do ar para que não haja escape do quarto de isolamento no momento de entrada e saída dos trabalhadores. O diferencial de pressão entre a antecâmara e o quarto de isolamento pode ser em torno de 7,5 a 15 Pa. A manutenção do diferencial de pressão demanda constante atenção em relação ao sistema de monitoramento do balanceamento do ar $(19,11)$.

No controle de fluxo de ar unidirecional em ambientes dos serviços de saúde, o movimento do ar deve ser de zonas limpas progressivamente para zonas de maior contaminação. Deve-se evitar turbulência do ar, uma vez que poderá causar mistura de agentes patogênicos no ar, aumentando o risco de infecções nosocomiais. Fatores que influenciam o padrão de fluxo unidirecional de ar são: estrutura física do ambiente, taxa de ventilação, interferência dos trabalhadores de saúde, instrumental cirúrgico, etc. O fluxo de ar unidirecional vertical (teto) e o horizontal (de parede para parede) são utilizados para remover, com eficácia, agentes patogênicos do ar do ambiente. No fluxo vertical, difusores de abastecimento de ar limpo em salas de cirurgia removem contaminantes dispersos pela equipe, sendo esse ar exaurido por grelhas laterais nas paredes próximas ao piso da sala. A direção do fluxo unidirecional de ar pode ser medida por um teste de fumaça utilizando-se tubos de fumaça química ou gelo seco (19). As salas de cirurgia para pacientes com TB requerem especial atenção na limpeza do ar exaurido devendo haver instalação de filtro absoluto no duto de saída de ar para não haver recirculação de ar contaminado. Nesses centros cirúrgicos para TB, devem ser estabelecidas medidas especiais de Biossegurança ambiental e de proteção respiratória por causa da classe de risco biológico do M. tuberculosis.

\section{CONSIDERAÇÕES FINAIS}

A qualidade do ar oferecido nos ambientes dos serviços de saúde e laboratórios merece atenção especial por parte dos gestores. O nível de poluentes atmosféricos e dos agentes de risco biológico e químico deve ser reduzido para evitar a propagação de infecções, principalmente a tuberculose, mas também as infecções respiratórias virais. Os diferentes ambientes dos serviços de saúde tornam-se únicos em razão dos desafios que apresentam. Entre eles destacam-se a ventilação e o condicionamento do ar, visto que sua função não é só fornecer conforto térmico aos profissionais e pacientes. A manutenção de um ambiente com qualidade do ar interior, no qual a concentração de partículas e agentes de risco biológico como M. tuberculosis seja reduzida, necessitará de controle, estudos integrados e do estabelecimento de protocolos e estratégias de Biossegurança. Dessa forma, é possível garantir um ambiente com ar interior de qualidade para tratamento e segurança dos pacientes, bem como para os profissionais de saúde. 


\section{REFERÊNCIAS}

1. Afonso MSM, Souza ACS, Tipple AFV, Machado EA, Lucas EA. Condicionamento de ar em salas de operação e controle de infecção: uma revisão. Rev Eletr Enf 8: 134-143, 2006.

2. Associação Brasileira de Normas Técnicas. ABNT. NBR 7256. Tratamento de ar em estabelecimentos assistenciais em saúde (EAS): Requisitos para projeto e execução das instalações. Rio de Janeiro, 2005.

3. Bagatin E, Kitamura S. História ocupacional. J bras Pneumol 32: S12-S16, 206.

4. Berlinguer G. Medicina e Política. Hucitec. São Paulo, 1978.

5. Brasil. Agência Nacional de Vigilância Sanitária. Resolução 176. Determina a publicação de orientação técnica elaborada por grupo técnico assessor, sobre padrões referenciais de qualidade do ar interior, em ambientes climatizados artificialmente de uso público e coletivo. Diário Oficial da União, 24 out. 2000.

6. Brasil. Agência Nacional de Vigilância Sanitária. Resolução- $R E n^{\circ}$. 9. Determina a publicação de orientação técnica elaborada por grupo técnico assessor, sobre Padrões Referenciais de Qualidade de Ar Interior, em ambientes climatizados artificialmente de uso público e coletivo. Diário Oficial da União, 16 jan. 2003.

7. Brasil. Ministério do Trabalho e Emprego. Portaria n. 485, de 11 nov. 2005. Aprova a Norma Regulamentadora n. 32 - segurança e saúde no trabalho em estabelecimentos de saúde. Diário Oficial da União. 16 nov. 2005.

8. Brasil. Ministério da Saúde. Secretaria de Vigilância em Saúde. Departamento de Vigilância Epidemiológica. Manual nacional de vigilância laboratorial da tuberculose e outras micobactérias. Brasília, 2008.

9. Brasil. Ministério da Saúde. Secretaria de Ciência, Tecnologia e Insumos Estratégicos. Departamento do Complexo Industrial e Inovação em Saúde. Classificação de risco dos agentes biológicos. Brasília, 2010.

10. Brasil. Ministério da Saúde. Secretaria de Ciência, Tecnologia e Insumos Estratégicos. Diretrizes gerais para o trabalho em contenção com agentes biológicos. Brasília, 2010.

11. Brito L. Tuberculose nosocomial medidas de controle de engenharia. Bol Pneumol Sanit 9: 33-50, 2001.

12. Brito RC, Zuim R, Carvalho RMG, Siqueira-Batista R, Bethlem EP, Bevilaqua AATP, Kritski A, Selig L. Recomendações da Assessoria de Pneumologia Sanitária do Estado do Rio de Janeiro para controle da tuberculose em hospitais gerais. Pulmão RJ 12: 169-173, 2003.

13. Centers for Disease Control and Prevention. Guidelines for Preventing the Transmission of Mycobacterium tuberculosis in Health-Care Settings, 2005. MMWR 54: 1-121, 2005.

14. Costa DC. Comentários sobre a tendência secular da tuberculose. Cad Saúde Pública 4: 398-406, 1988.

15. Gonçalves H. A tuberculose ao longo dos tempos. Hist Cienc Saude-Manguinhos 7: 305-327, 2000.

16. Graça L, Henriques AI. Florence Nigthingale e Ethel Fenwick: da ocupação à profissão de enfermagem. Disponível em: http://www.ensp.unl.pt/lgraca/textos63.html. Acesso em 24/02/2012.

17. Jensen PA, Lambert LA, Iademarco MF, Ridzon R. Guidelines for preventing the transmission of Mycobacterium tuberculosis in health-care settings. MMWR Recomm Rep 54: 1-141, 2005

18. Lacerda RA, Egry EY. As infecções hospitalares e sua relação com o desenvolvimento da assistência hospitalar: reflexões para análise de suas práticas atuais de controle. Rev Latino-Am Enfermagem 5: 13-23, 1997.

19. Leung M, Chan AHS. Control and management of hospital indoor air quality. Med Sci Monit 12: SR17-23, 2006.

20. Lima MM. HIV/tuberculosis co-infection: a request for a better surveillance. Rev Saúde Públ 31: 217-220, 1997.

21. Lopes OG. Conforto térmico e qualidade do ar em ambientes. 2004. Disponível em: http://cursos. unisanta.br/mecanica/polari/ct-og.pdf. Acesso em 27/02/2012. 
22. Maciel ELN, Prado TN, Fávero JL, Moreira TR, Dietze R. Tuberculose em profissionais de saúde: um novo olhar sobre um antigo problema. J Bras Pneumol 35: 83-90, 2009.

23. Malasky C, Jordan T, Potuslski F, Reichman LB. Occupational tuberculosis infections among pulmonary physicians in training. Am Rev Respir Dis 142: 505-507, 1990.

24. Menzies D, Fanning A, Yuan L, Fitzgerald M. Tuberculosis among health care workers. $N$ Engl $J$ Med 332: 92-98, 1995.

25. Moon P. John Oxford: “A pandemia não acabou”. Época 630: 11/06/2010. Disponível em: http:// revistaepoca.globo.com/Revista/Epoca/0,EMI147048-15257,00-JOHN+OXFORD+A+PANDE MIA+AINDA+NAO+ACABOU.html. Acesso em 22/02/2012.

26. National Institute for Occupational Safety and Health. Tuberculosis. Disponível em: http://www. cdc.gov/niosh/topics/tb/. Acesso em: 23/02/2012.

27. Nobre LCC. Estudo referente à utilização da Classificação Brasileira de Ocupações (CBO) e da Classificação Nacional de Atividades Econômicas (CNAE) nos Sistemas de Informação em Saúde. Organização Pan-Americana da Saúde. Brasília, 2002.

28. Pandemia no ar. Pesquisa investiga cobertura da influenza A no jornal nacional. RADIS Comunicação em saúde 93: 2, 2010.

29. Rocha JL, Dalcolmo MP, Borga L, Fedele D, Marques MG . Tuberculose multirresistente. Pulmão RJ 17: 27-32, 2008.

30. Santos BMO. Monitoramento da colonização pelo Staphylococcus aureus em alunos de um curso de auxiliar de enfermagem durante a formação profissional. Rev Latino-Am Enfermagem 8: 67-73, 2000.

31. Snider Jr DE, La Montagne JR. The neglected global tuberculosis problem: a report of the 1992 World Congress on Tuberculosis. J Infect Dis 169: 1189-1196, 1994.

32. World Health Organization - WHO. Policy on TB Infection Control in Health-Cares Facilities. Congregate Settings and Households. Geneva, 2009. 